\title{
A Case of Fatal Food-borne Septicemia: Can Family Physicians Provide Prevention?
}

Gaines Martin, MD, PhD, Anna M. Wright, MD, and Kay Banakarim, $M D$

Background: Vibrio vulnificus, a common bacteria found in undercooked seafood and seawater, is the leading cause of food-borne death in Florida. Fatal cases of $\boldsymbol{V}$ vulnificus infection have also been reported in most states.

Metbods: The literature was searched using the key words "Vibrio vulnificus," "septicemia," "wound infections," "seafood," "immunocompromise," and "patient education." A case of fatal V vulnificus septicemia is described.

Results and Conclusions: $V$ vulnificus, part of the natural flora of temperate coastal waters and one of the most abundant microorganisms found in seawater, has been isolated from waters off the Gulf, Pacific, and Atlantic coasts of the United States. Infections in noncoastal regions have been traced to consumption of seafood derived from Gulf Coast waters. Seawater exposure and consumption of inadequately cooked seafood are routes most commonly associated with $V$ vulnificus infection. Exposure to $V$ vulnificus is life-threatening for chronically ill or immunocompromised patients, who are most likely to develop fatal septicemia. Currently a combination of doxycycline and intravenous ceftazidime is recommended treatment. Mortality rates from V vulnificus continue to be high in immunocompromised patients. Family physicians can help prevent this outcome by counseling high-risk patients.(J Am Board Fam Pract 2000;13:197-200.)

Vibrio vulnificus, a common bacteria found in seawater and undercooked seafood, is the leading cause of food-borne mortality in Florida. Fatalities from $V$ vulnificus infection, however, have been reported in most states as a result of the popularity and availability of shellfish. Chronically ill or immunocompromised patients are predisposed to develop fulminant, fatal $V$ vulnificus septicemia. Mortality rates from Vibrio sepsis remain high, even with prompt diagnosis and appropriate treatment. A key to preventing deaths from $V$ vulnificus infection is primary prevention by family physicians to recognize and counsel high-risk patients. ${ }^{1}$

\section{Methods}

MEDLINE was searched using the key words "Vibrio vulnificus," "septicemia," "wound infections," "seafood," "immunocompromise," and "patient education." A case of fatal $V$ vulnificus septicemia in a 65-year-old woman with cirrhosis of the liver and end-stage renal disease is reported.

Submitted, revised, 15 September 1999.

From the Department of Community Health and Family Medicine (GM, AMW, KB), University of Florida Health Science Center, Jacksonville. Address reprint requests to Anna $M$. Wright, MD, University Family Practice Center, 1255-B Lila St, Jacksonville, Fl 32208.

\section{Case Report}

A 65-year-old woman came to the emergency department complaining of acute onset of right calf pain lasting several hours. She denied trauma or other complaints. Her medical history was notable for cirrhosis of the liver and end-stage renal disease requiring peritoneal dialysis.

At her physical examination her temperature was $100.6^{\circ} \mathrm{F}$, pulse 60 beats per minute, respirations $20 / \mathrm{min}$, and blood pressure $65 / 48 \mathrm{~mm} \mathrm{Hg}$, and she had normal oxygen saturation. The patient was alert, oriented, and in mild distress secondary to pain. Findings of the patient's heart and lung examination were unremarkable, her abdomen was soft, and nontender, and the dialysis catheter site showed no sign of infection. There was notable right calf tenderness to palpation, but no edema, erythema, rash, or skin lesions. The patient received intravenous saline and had prompt improvement in her blood pressure to $108 / 67 \mathrm{~mm} \mathrm{Hg}$, and she was admitted for further evaluation.

Initial laboratory results were hemoglobin 9.4 $\mathrm{g} / \mathrm{dL}$, hematocrit $27.7 \%$, platelet count 158,000 / $\mathrm{mL}$, and white blood cell count $5900 / \mu \mathrm{L}$ with a left shift. A Gram stain of the peritoneal fluid was negative. Doppler sonography of her lower extremities showed no evidence of deep venous thrombo- 
phlebitis. Findings from a chest radiograph and an electrocardiogram were unremarkable.

The patient remained stable but continued to complain of calf pain that seemed out of proportion to her examination findings. Approximately 24 hours after her admission, she suddenly developed respiratory distress, hypotension, and mental status deterioration. At this time her pulse was 97 beats per minute, respirations $40 / \mathrm{min}$, and blood pressure $80 / 44 \mathrm{~mm} \mathrm{Hg}$, and her oxygen saturation was normal. Arterial blood gases showed a severe metabolic acidosis with a $\mathrm{pH}$ of 6.9 . The patient was transferred immediately to the intensive care unit. In the intensive care unit, a nasogastric lavage showed coffee-ground gastric material. Notable laboratory results at this time were hemoglobin 4.0 $\mathrm{g} / \mathrm{dL}$, a positive D-dimer test, and increased levels of fibrin split products. She was aggressively treated for gastrointestinal hemorrhage, hypotension, and disseminated intravascular coagulopathy. She remained hypotensive and died approximately after 32 hours of hospitalization.

The day after her death, blood cultures from samples taken when she was admitted to the hospital grew $V$ vulnificus. Later it was learned she had consumed crabmeat the day of admission.

\section{Discussion}

$V$ vulnificus, a gram-negative bacillus that thrives in brackish water along subtropical coastal areas, was first described in 1979 and today is the leading cause of food-borne mortality in Florida. ${ }^{1}$ The number of reported cases continues to increase yearly. Between 1981 and 1993, V vulnificus was responsible for $80 \%$ of reported food-borne deaths in Florida, making it the most common food-borne cause of death in Florida during that period. ${ }^{2} V$ vulnificus is part of the Vibrio genus, which includes the well-known pathogens $V$ cbolerae and $V$ parabaemolyticus. ${ }^{1}$ In the United States, $V$ vulnificus has been isolated from waters off the Gulf Coast and the Pacific and Atlantic coasts of the United States, and off the coast of Hawaii. ${ }^{3}$ Nearly $50 \%$ of oyster lots from Florida estuaries are culture-positive for $V$ vulnificus, making it part of the natural flora of Florida coastal waters and one of the most abundant microorganisms found in seawater. ${ }^{4,5}$. Fatalities caused by this organism have occurred in most states along the Gulf Coast, and in California, Utah, Kentucky, Oklahoma, and Arkansas. Infec-
Table 1. Findings Associated With Vibrio vulnificus Infection.

\begin{tabular}{ll}
\hline Abdominal cramps & Leg pain \\
Bulla formation & Localized cellulitis \\
Chills & Male sex \\
Diarrhea & Nausea \\
Fever & Shock \\
Hypotension & Vomiting \\
\hline
\end{tabular}

Adapted from Shapiro et $\mathrm{al}^{8}$ and Kumamoto and Vukich. ${ }^{9}$

tions in noncoastal regions have been traced to consumption of seafood derived from Gulf Coast waters.

Exposure to $V$ vulnificus results in several different outcomes based on the degree of immunocompromise of affected persons. ${ }^{6} \mathrm{~V}$ vulnificus infection has three distinct clinical syndromes: gastroenteritis, wound infection, and primary septicemia. $\mathrm{Pa}$ tients with liver disease or immunocompromised states are at risk of developing severe infections, whereas healthy persons experience mild illness. Seawater exposure and consumption of inadequately cooked seafood are routes most commonly associated with $V$ vulnificus infection. Immunocompetent persons can develop a self-limited gastroenteritis after ingestion of raw or undercooked oysters, crabs, shrimp, or mussels. Skin infections are caused by penetration of the organism through preexisting skin lesions or through lesions incurred during seawater-related activities, such as cleaning fish or shucking oysters. Often no identifiable abrasions or lacerations are discovered at the time of hospital admission. Skin infections, however, can progress to necrotizing fasciitis and require surgical intervention. ${ }^{?}$

In immunocompromised hosts, exposure to $V$ vulnificus is associated with life-threatening primary septicemia, either through the gastrointestinal tract or through wounds. Onset of severe illness with fulminant septicemia is rapid. Symptoms include fever, chills, lower extremity pain, nausea, vomiting, and bulla formation, as well as others listed in Table 1.,9 In immunocompromised patients, $V$ vulnificus infection is associated with a greater than $40 \%$ mortality overall, and nearly $100 \%$ mortality when untreated or when sepsis develops. In cases of most fatal infections, patients had preexisting risk factors listed in Table $2 .^{8,10}$ The pathophysiologic basis underlying why liver disease is a serious risk factor for infection is unclear. 
Table 2. Conditions Leading to Increased Risk of Fatal Vibrio vulnificus Infection.

\begin{tabular}{ll}
\hline Alcoholic liver disease & Hepatitis B \\
Cancer & Hepatitis C \\
Chemotherapy & Hypochlorhydria \\
Chronic corticosteroid use & Immunodeficiency syndromes \\
Chronic renal disease & Jaundice \\
Cirrhosis & Liver transplant \\
Diabetes mellitus & Sickle cell disease \\
Hemochromatosis & Splenectomy \\
Hemolytic anemia & Thrombocytopenia \\
\hline
\end{tabular}

Adapted from Shapiro et $\mathbf{a l}^{8}$ and Kumamoto and Vukich. ${ }^{9}$

The outcome of $V$ vulnificus sepsis infection in immunocompromised patients is poor because of the rapid course of the illness and its elusive clinical signs and symptoms. Debate continues about the appropriate antimicrobial therapy. No single regimen has been shown to be superior, as evidenced by the continued high case fatality rate. Mortality has been reported by some authors to be $60 \%$ or greater, even though many antimicrobials have been shown to be effective at preventing growth of the organism in vitro. Currently a combination of intravenous or oral doxycycline, $100 \mathrm{mg}$ twice a day, and intravenous ceftazidime, $2.0 \mathrm{~g}$ every 8 hours, is recommended. Other treatment options are listed in Table 3.9 Because treatment is often initiated too late or is incapable of overcoming the infection once the patient is symptomatic, reducing exposure of immunocompromised patients to the organism is the best means of preventing fatal $V$ vulnificus infections.

Primary prevention of disease involves realizing which patients are at risk of developing specific illnesses and informing them how to avoid exposure. Because of the relatively low incidence of $V$ vulnificus infections, 0.4 to 1.9 reported cases per 1 million people, many care providers are unaware of the risk to their patients. ${ }^{11}$ Most patients who are at risk of developing $V$ vulnificus infection are readily

\section{Table 3. Therapy for Vibrio vulnificus Infections.}

1. Intravenous ceftazidime $2.0 \mathrm{~g}$ every $8 \mathrm{~h}$, and intravenous or oral doxycycline $100 \mathrm{mg}$ twice a day

2. Supportive care (for shock)

3. Active toxin removal (experimental)

4. Surgical debridement of wounds

Adapted from Kumamoto and Vukich. ${ }^{9}$
Table 4. Modes of Prevention of Infection Directed at Patients at Risk.

1. Patient education by family physicians and internists

2. Patient education by gastroenterologists, nephrologists, and oncologists

3. Public warnings in restaurants

4. Information packets and pamphlets in medical offices and dialysis units

5. Public announcements and advertisements

Adapted from Mouzin et al. ${ }^{12}$

recognizable, as shown in Table 2 . No additive effect of individual risk factors has been reported in the literature, but a compilation of incidence of infection based on preexisting illnesses and conditions has been established. ${ }^{8}$ Because $V$ vulnificus infection is a preventable disease, it is important to use preventive methods designed to target patients at risk and to develop effective methods to reduce this risk. A variety of patient education approaches for prevention of $V$ vulnificus infection have been suggested, as shown in Table $4 .{ }^{10}$ In 1990 , California became the first state to require restaurants that serve raw oysters to warn customers of their associated health risks. Currently, Alabama, California, Florida, Louisiana, and Mississippi require restaurants serving raw oysters to post a placard warning persons with liver disease and immunosuppressed states to avoid eating these foods. In one survey only $71 \%$ of restaurants were compliant with this law. ${ }^{12}$ Efforts to publicize the risks of $V$ vulnificus infection to health care professionals and the public have been initiated but have not been widespread. Thus far, the incidence of infection remains unchanged.

Patients at risk for $V$ vulnificus infection should receive specific counseling from their family phy-

Table 5. Physician Advice for Immunocompromised Patients.

1. Avoid raw seafood, and ensure that seafood eaten in restaurants and at home is adequately cooked

2. Fry, bake, steam, or boil oysters 4-9 min or until plump. Boil shrimp until shells turn pink and meat is cooked in middle (3-5 min). Fish is adequately cooked when the thickest part is opaque. Seafood should generally be cooked to an internal temperature of $145^{\circ} \mathrm{F}$ or $63^{\circ} \mathrm{C}$

3. Avoid exposure to ocean water in summer months or along coastal regions in the southeastern United States

4. Inform any health care professional caring for you about your medical condition, and provide a list of medications

Adapted from Department of Health and Human Services. ${ }^{13}$ 
sicians, as slight modifications in diet and activities are key to prevention (Table 5). First, patients should be advised to avoid raw oysters, clams, shrimp, and fish because of the high incidence of $V$ vulnificus in these foods. They should try to eat well-cooked seafood, being aware of the risks of eating undercooked seafood. Avoidance of seafood in summer months would also be prudent. Second, patients should avoid exposure to ocean water or ocean products in the summer or in warm climates, such as those found in the southern United States, because infection is more likely to occur in these areas. Third, patients should be advised to inform health care professionals caring for them of all underlying medical conditions and medications. A patient education brochure entitled "If You Eat Raw Oysters You Need to Know ..." published by the Food and Drug Administration is available for physicians and patients by calling the FDA Seafood hotline, 800-FDA-4010, or contacting the FDA through its Web site at www.fda.gov. ${ }^{13}$

As primary care providers for many patients at high risk for $V$ vulnificus infection, family physicians have a unique opportunity to help reduce morbidity and mortality in this patient group. Through increased awareness and patient education, family physicians can provide primary prevention to atrisk patients. The family physician's role in prevention of $V$ vulnificus infection in at-risk patients is an important one.

\section{References}

1. Hlady WG, Klontz KC. The epidemiology of Vibrio infections in Florida, 1981-1993. J Infect Dis 1996; 73:1176-83.

2. Kizer K. Vibrio vulnificus hazard in patients with liver disease. West J Med 1994;161:64-5.
3. Wright AC, Hill RT, Johnson FA, Rogham MC, Colwell RR, Morris JG Jr. Distribution of Vibrio vulnificus in the Chesapeake Bay. Appl Environ Microbiol 1996;62:717-24.

4. Hlady WG, Mullen RC, Hopkins RS. Vibrio vulnificus from raw oysters. Leading cause of reported deaths from foodborne illness in Florida. J Fla Med Assoc 1993;80:536-8.

5. Vibrio vulnificus infections associated with raw oyster consumption-Florida, 1981-1992. MMWR Morb Mortal Wkly Rep 1993;42:405-7.

6. Farmer JJ 3d. Vibrio ("Beneckea") vulnificus, the bacterium associated with sepsis, septicemia, and the sea. Lancet 1979;2:903.

7. Stabellini N, Camerani A, Lambertini D, et al. Fatal sepsis from Vibrio vulnificus in a hemodialyzed patient. Nephron 1998;78:221-4.

8. Shapiro RL, Altekruse S, Hutwagner L, et al. The role of Gulf Coast oysters harvested in warmer months in Vibrio vulnificus infections in the United States, 1988-1996. Vibrio Working Group. J Infect Dis 1998;178:752-59.

9. Kumamoto KS, Vukich DJ. Clinical infections of Vibrio vulnificus: a case report and review of the literature. J Emerg Med 1998;16:61-6.

10. Mouzin E, Mascola L, Tormey MP, Dassey DE. Prevention of Vibrio vulnificus infections. Assessment of regulatory educational strategies. JAMA 1997;278: 576-8.

11. Vollberg CM, Herrera JL. Vibrio vulnificus infection: an important cause of septicemia in patients with cirrhosis. South Med J 1997;90:1040-2.

12. Gholami P, Lew SQ, Klontz KC. Raw shellfish consumption among renal disease patients. A risk factor for severe Vibrio vulnificus infection. Am J Prev Med 1998;15:243-5.

13. If you eat raw oysters, you need to know.... Rockville, Md: Office of Public Affairs, Food and Drug Administration, 1995. [DHHS publication no. (FDA) 95-2293.] 